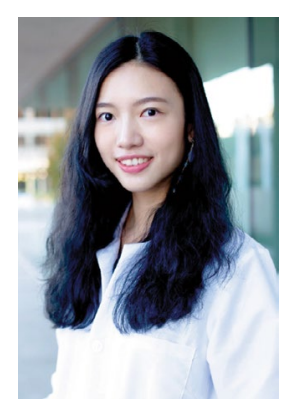

Credit: Shiley eye institute

\title{
Asian healthcare workers in the COVID-19 pandemic: a long road to recovery
}

\author{
Asian healthcare workers have experienced racism during the COVID-19 pandemic, including \\ from the medical community, with potentially long-term consequences for those affected.
}

A lmost one and a half years ago, the first case of COVID-19 was confirmed in Wuhan, China, followed by a state of global health emergency due to a worldwide pandemic. Effective vaccines against COVID-19 were finally released, and over $40 \%$ of the US population has now been fully vaccinated. The battle of the United States versus COVID-19 seems to have reached the mitigation phase. However, for many living in this country, the detrimental effects of this virus may still be ongoing.

Long before the pandemic, the Asian population in the United States had been viewed and treated as the physical embodiment of foreignness and disease, and this stigma does not disappear because you are a medical worker wearing a white coat. As the stress levels of society elevated with the outbreak of a China-originated virus, the increase in negative bias against the Asian population was perhaps not surprising.

When I first moved to the United States to advance my career, it was right at the peak of the pandemic. News of racist assaults and ignorant attacks against Asians were broadcast, and I can recall some headlines saying 'the assaults are spreading faster than the coronavirus'. Although I live in one of the most Asian-friendly states, in California, it was not easy to reassure myself and others about my personal safety.

While many can practice social isolation by working from home, most healthcare workers are still meeting people of all ethnic backgrounds and personal values. Born and raised in a country with limited understanding about racial discrimination, I was prepared for possible hostility from people of different skin colors. "To some, everyone with yellow skin either has COVID19 , or should be partially responsible for the impact of COVID-19 in this country," said one of my Asian American friends. Were Asian healthcare workers treated aggressively by racist patients? Yes. Were they teased disrespectfully on subjects about COVID-19?
Of course. However, because of our jobs as healthcare workers, we are used to ignoring such comments and suppressing our feelings when we encounter discrimination.

I later realized that racism does not come only from patients. Occasionally, discrimination against Asians, including microaggressions, can be observed within the medical community. These microaggressions may be an anonymous comment in an online physician forum, a careless statement recorded in an academic interview, an ignorant joke told by some hospital workers passing by, or even an unconscious act of a colleague. Physical attacks are not common in our work environments, but emotional and verbal aggressions can be seen in all forms and from all sources. Are these comments repeated when people leave work and interact with others in non-medical professions? We cannot know. Nevertheless, with the public influence of healthcare workers, due to their professional roles, even the least intended discriminative language may worsen the overall effect on society. Sometimes I would tell myself, "You may need to fight not only the coronavirus, but also the racism in your working environment." In the time of COVID-19, this fear of Asian healthcare workers can be easily learned.

It is undeniable that Asian healthcare workers have suffered from an aggravated negative bias because of the COVID-19 pandemic. However, I have also learned to appreciate the opposite side of this; the pursuit of diversity and equality is also a shared goal for many in this country. Owing to my cultural background, in the past I was unaware that not being discriminated against on the basis of your skin color is essential for good mental health. Now I am grateful that I work in a department that has long focused on diversity and equality issues. I have also witnessed many non-Asian medical workers using their voices to help eliminate anti-Asian sentiments. Seeing the efforts of colleagues and strangers, I feel encouraged to speak up for other Asian healthcare workers who may or may not be lucky enough to feel safe, secure and respected during the COVID-19 pandemic.

With the rapid distribution of vaccines in the United States, it is estimated that normality may resume imminently. Beyond the control of COVID-19, it is unclear what other aspects of normality should be expected. The balance between public health and infection? The resumption of social activities? Or the recovery of a thriving economy? In addition to the number of infections and hospitalizations, the complications of aggravated racism in certain populations must be considered. Recovery from the social and psychological aspects of the COVID-19 pandemic will take longer than recovery from the disease. This will require medical workers to attend to the racism-related experiences and mental health needs of not only their patients but also their co-workers.

COVID-19 swept through and shook the United States on multiple levels. The pandemic revealed not only the weakness of the human immune system and the US healthcare system but also the underestimated extent of racial discrimination in society and within the medical community. COVID-19 has taught a lesson that we are grateful for, but also fearful of. For many Asian healthcare workers, the negative experience we have gone through may not be cured or healed easily. The road to recovery is long, and for everyone working in the medical field, this is one important goal we should fight together for.

\section{Jo-Hsuan Wu (1D}

Shiley Eye Institute and Viterbi Family Department of Ophthalmology, University of California, San Diego, CA, USA.

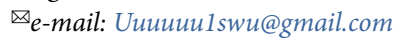

Published online: 28 June 2021

https://doi.org/10.1038/s41591-021-01424-4

Competing interests

The author declares no competing interests. 